\title{
Secondary prevention after ischaemic stroke: the ASPIRE-S study
}

\author{
Linda Brewer ${ }^{1 *}$, Lisa Mellon², Patricia Hall², Eamon Dolan ${ }^{3}$, Frances Horgan ${ }^{4}$, Emer Shelley ${ }^{5}$, Anne Hickey ${ }^{2}$, \\ David Williams', on behalf of the ASPIRE-S Study Group
}

\begin{abstract}
Background: Survivors of ischaemic stroke (IS) are at high-risk for future vascular events. Comprehensive information on the adequacy of secondary prevention after IS is lacking despite the knowledge that appropriate secondary prevention improves long-term patient outcomes. ASPIRE-S (Action on Secondary Prevention Interventions and Rehabilitation in Stroke) aimed to prospectively assess secondary prevention in patients 6 months following IS.

Methods: Consenting patients admitted with IS to three Dublin hospitals were recruited over 1 year, from October 2011. At 6 months post IS a comprehensive assessment was completed, modelled on the EUROASPIRE protocol for evaluation of the adequacy of secondary prevention in post-discharge cardiac patients. This assessment included measurements of blood pressure, body mass index and fasting lipid and glucose profiles. Secondary preventive medications and smoking status were also documented.

Results: Three hundred two patients (58\% male) participated, of whom $256(85 \%)$ were followed-up at 6 months. Mean age was 69 years (range 22-95). At follow-up, $68 \%$ of patients had a BMI $>25 \mathrm{~kg} / \mathrm{m}^{2}$ and $16.4 \%$ were still smoking. Almost two-thirds (63.4\%) had a blood pressure >140/90 and $23 \%$ had low-density-lipoprotein $>2.5$ mmol/L. $28 \%$ of diabetic patients had HbA1c $\geq 7 \%$. Ninety seven percent of patients were on anti-platelet and/or anticoagulant therapy. Of those with atrial fibrillation, $82 \%$ were anti-coagulated (mean INR of 2.4). Ninety-five percent were on lipid-lowering therapy and three-quarters were on anti-hypertensive therapy.
\end{abstract}

Conclusion: This prospective multi-centre survey of IS patients demonstrated a high prevalence of remaining modifiable risk factors at 6 months post stroke, despite the widespread prescription of secondary preventive medications. There is scope to improve preventive measures after IS (in particular blood pressure) by incorporating evidence-based guidelines into quality assurance cycles in stroke care.

Keywords: Ischaemic stroke, Secondary prevention, Risk factors

\section{Background}

Stroke is a leading cause of death and disability resulting in substantial personal and healthcare costs. Although age-standardised rates of stroke mortality have decreased, the absolute number of people suffering stroke annually, stroke survivors and overall stroke burden are increasing [1]. Approximately $30 \%$ of strokes occur in individuals with a previous stroke and $50 \%$ occur in those with previous vascular events of any kind [2]. High recurrence rates emphasize the importance of effective

\footnotetext{
* Correspondence: lindabrewer@beaumont.ie

'Department of Geriatric \& Stroke Medicine, Royal College of Surgeons in Ireland (RCSI), Beaumont Hospital, Dublin 9, Ireland

Full list of author information is available at the end of the article
}

preventive strategies and many population-based studies have reported strong associations between numerous cardiovascular risk factors and future stroke risk [2-4]. These findings have informed the development of evidencebased guidelines on stroke prevention $[5,6]$ which aim to improve stroke outcomes.

The EUROASPIRE surveys [7-9] drew attention to the continuing gap between standards set in guidelines on secondary cardiovascular disease prevention (in patients with coronary heart disease) and results achieved in clinical practice. More recently, a stroke-specific module retrospectively added to EUROASPIRE III concluded that risk factor control after IS also requires improvement [10]. 
We report a prospective, multi-centre study which assessed the extent to which evidence-based care was provided to a sample of Irish patients admitted with IS, as part of the Action on Secondary Prevention Interventions and Rehabilitation in Stroke (ASPIRE-S) study. This study reviewed three components of IS care following discharge, including the adequacy of secondary prevention, delivery of rehabilitative care and assessment of ongoing rehabilitative needs. This analysis focuses on the adequacy of secondary prevention at 6 months in the ASPIRE-S cohort.

\section{Methods}

\section{Study hospitals and patient recruitment}

Patients aged 18 years and over with IS admitted to three study hospitals were recruited between October 2011 and September 2012. Ethical approval was granted from the Medical Research Ethics Committees of participating hospitals, Beaumont Hospital (BH), Mater Misericordiae University Hospital (MMUH) and Connolly Hospital (CHB). All three study hospitals are university-affiliated teaching hospitals located in suburban $(\mathrm{BH}, \mathrm{CHB})$ and city (MMUH) North Dublin locations, serving a combined catchment area of over 750,000 people. The stroke population in North Dublin has previously been characterised by the North Dublin Population Stroke Study group (NDPSS) [11, 12], which reported that over $90 \%$ of acute stroke cases are treated in an acute hospital. For the ASPIRE-S study, hospital-based case ascertainment was performed and a representative sample of patients with IS was sought through review of daily admissions via emergency departments and regular review of stroke consult lists in each hospital. In advance of the commencement of this study, the ASPIRE-S study team met with the stroke teams at each site to explain the study inclusion and exclusion criteria. Patients were considered eligible if they had a World Health Organisation (WHO) defined IS to include ICD-10 code 163 (cerebral infarction), including subcategories and were medically well enough for participation.

\section{Data collection at baseline}

At recruitment, baseline details (including demographic details, stroke subtypes using TOAST [13] and Bamford [14] classifications and stroke risk factors) were collected during a short structured bedside interview. For patients who presented with severe stroke, their progress was monitored over time and their participation was requested once they were considered medically stable. Every effort was made to include patients with severe strokes, to avoid selection bias in favour of milder strokes. All eligible patients were either approached in person or (if already discharged) were phoned by a member of the research team to discuss the study and to obtain consent. Where capacity was inadequate, consent to participate was obtained from the next of kin.

\section{Patient interview and examination at six months}

At 6 months post-stroke, patients were contacted for follow-up at which point a detailed interview and examination were carried out, using standardised methods and instruments. Most patients were reviewed in their own home by a trained member of the research team, with a minority of patients returning to a Clinical Research Centre for assessment. All patients were requested to fast from $8 \mathrm{pm}$ on the preceding night for the measurement of fasting blood tests at the visit. For the purpose of this study, blood results were compared with targets outlined in the European Guidelines on cardiovascular disease prevention in clinical practice (version 2012) [15] and (for diabetes) the American Diabetes Association 2013 Guidelines [16].

\section{Anthropometrical and physiological measurements Blood pressure}

Following the measurement of arm circumference and the application of an appropriately sized cuff, patients were asked to sit comfortably with their arm relaxed and at heart level. Blood pressure (BP) was measured using a digital OMRON M6 (Intellisense ${ }^{\mathrm{TM}}$ ) Dual Check System. To allow for comparison with EUROASPIRE [7], the first BP measurement was recorded from the right arm, where possible. To assess for an important difference in BP between arms, we also recorded BP from the left arm. Twenty-four hour control of BP was assessed by applying a SpaceLabs 90207 monitor (SpaceLabs Inc, Wokingham, Berkshire, $\mathrm{UK}$ ) to the arm with the highest $\mathrm{BP}$ reading (where possible). This measured BP every $30 \mathrm{~min}$ by day ( $8 \mathrm{am}$ to $10 \mathrm{pm}$ ) and every $60 \mathrm{~min}$ overnight (10 pm to $8 \mathrm{am}$ ).

\section{Waist circumference/BMI}

Waist circumference was measured by applying a standard flexible (Farla Medical, UK) measuring tape circumferentially at the level of iliac crest and was compared with WHO-defined targets (male target <94 cm; female target $<80 \mathrm{~cm}$ ). Body weight was measured to the nearest kilogram using a calibrated (EKS International, France) mechanical weighing scales. Height was measured as the maximum distance from the floor to the vertex of the head and was recorded to the nearest centimetre. Body mass index (BMI) was calculated using the formulation weight $(\mathrm{kg}) /(\text { height }(\mathrm{m}))^{2}$ and scores were categorised according to Centres for Disease Control and Prevention criteria [17].

\section{Fasting blood tests}

Patients had venous blood drawn to measure fasting glucose (FG) and full lipid profiles including total cholesterol 
(TC), low density lipoprotein (LDL), high density lipoprotein (HDL) and triglycerides (TG). Glycosylated haemoglobin (HbA1c) was measured in diabetic patients. All blood samples were processed according to local protocols governed by the Clinical Directorate of Laboratory Medicine. For patients on warfarin, recent international normalised ratio (INR) records (using patient-held booklets) were reviewed to assess the adequacy of anti-coagulation.

\section{Secondary preventive medications}

A full list of medications was recorded by reviewing the patient's most recent prescription, pillbox or medication containers.

\section{Study governance and quality assurance}

Study recruitment, follow-up and data management took place under the supervision of the study steering committee. To ensure uniformity of method the first 106 month visits were conducted in pairs (two of three trained researchers) and thereafter all three researchers conducted the follow-up assessments in the same manner, using identical equipment. At regular intervals throughout the study period, these assessments were conducted in pairs to assure ongoing quality and uniformity of data collection.

\section{Dataset and analysis}

Descriptive statistics summarised and described the main findings, including percentages for categorical variables, and means and standard deviations (SD) for quantitative data. Comparisons between subgroups were performed using chi-squared analysis. Significance was calculated at a level of $p<0.05$. Data were analysed using STATA (StataCorp 2013, Texas).

\section{Results}

Baseline characteristics

Three hundred two patients (mean age 69.1 years, standard deviation 12.8, range 21.8-94.9) with acute IS agreed to participate. Demographic characteristics, stroke subtype and risk factors are outlined in Table 1. Overall, patients had a mean number of three risk factors (SD 1.7; range 0-8), with almost all patients (240; $94 \%)$ having at least one risk factor for stroke at baseline.

Of 302 recruited patients, 46 patients (15\%) were unavailable for assessment at 6 months. Reasons for non-participation included patient refusal $(n=22)$, death $(n=9)$, intercurrent serious illness $(n=6)$, uncontactable $(n=5)$ and final diagnosis not stroke $(n=4)$. The remaining 256 patients participated in the follow-up assessment. There were no documented cases of recurrent stroke in the 6 month follow-up period for the 256 patients included.

\section{Risk factors at six months \\ Fasting blood results}

Blood was drawn from 232 patients $(90.5 \%$ of the cohort) to measure (FG) and lipid profiles. Blood was not drawn due to patient refusal, unsuccessful phlebotomy or failure to fast appropriately. Mean glucose level was $5.4 \mathrm{mmol} / \mathrm{l}$ (SD,1.35). HbA1c was measured in $91 \%$ $(n=46)$ of diabetic patients and the mean level was $6.7 \%$ (range 5.4-10.5; SD 0.99). Over one-quarter (28\%) of diabetic patients had an HbA1c $\geq 7 \%$ and $29.4 \%$ had FG $\geq 7 \mathrm{mmol} / \mathrm{l}$. Amongst non-diabetic patients, $1.5 \%$ had a glucose level $\geq 7.0 \mathrm{mmol} / \mathrm{L}$ (diabetic range), $3.9 \%$ had a result between 6.1 and $6.9 \mathrm{mmol} / \mathrm{L}$ (impaired fasting glucose range - Europe) and $9.6 \%$ had a result between 5.6 and $6.9 \mathrm{mmol} / \mathrm{L}$ (impaired fasting glucose range - American Diabetes Association 2013) [18]. Mean lipid values (with

Table 1 Baseline demographic characteristics, stroke subtypes and risk factors $(N=302)$

\begin{tabular}{|c|c|c|c|c|c|}
\hline Demographic details & N (\%) & Stroke subtype & N (\%) & Risk factor & N (\%) \\
\hline Age & & Bamford & & Hypertension & $176(57)$ \\
\hline$<65$ & $103(34)$ & PACS & $117(38.7)$ & Hypercholesterolemia & $135(46.9)$ \\
\hline \multirow[t]{2}{*}{$\geq 65$} & $199(66)$ & LACS & $81(26.8)$ & Atrial fibrillation & $120(38.3)$ \\
\hline & & POCS & $80(26.5)$ & Heart disease & $91(29.3)$ \\
\hline Gender & & TACS & $16(5.3)$ & Current smoking & $84(28.1)$ \\
\hline Male & $173(57.3)$ & Unclassifiable & $8(2.7)$ & Previous TIA/stroke & $76(25)$ \\
\hline \multirow[t]{2}{*}{ Female } & $129(42.7)$ & & & Diabetes & $60(19.9)$ \\
\hline & & TOAST & & Past smoking & $53(17.6)$ \\
\hline Function (mRS) & & Cardioembolism & $121(40.1)$ & Carotid disease & $50(16)$ \\
\hline$\leq 2$ & $156(51.7)$ & Undetermined aetiology & $84(27.8)$ & Alcohol excess & $44(14.5)$ \\
\hline \multirow[t]{3}{*}{$>3$} & $146(48.3)$ & Large vessel atherosclerosis & $51(16.9)$ & Depression & $24(8.2)$ \\
\hline & & Small vessel disease & $33(10.9)$ & Anxiety & $20(6.6)$ \\
\hline & & Other determined aetiology & $13(4.3)$ & & \\
\hline
\end{tabular}


SD) were TC $4 \mathrm{mmol} / \mathrm{l}$ (0.99), LDL $2.1 \mathrm{mmol} / \mathrm{l}$ (0.88), HDL $1.3 \mathrm{mmol} / \mathrm{l}(0.37)$ and TG $1.4 \mathrm{mmol} / \mathrm{l}(0.72)$. The proportion of patients with fasting lipid results at target is illustrated in Fig. 1.

\section{Blood pressure}

Office blood pressure The mean right arm systolic BP was $148 \mathrm{mmHg}$ (range 92-207; SD 22.2) and the mean right arm diastolic BP was $81 \mathrm{mmHg}$ (range 40-119; SD 12.8). Most patients did not reach recommended international targets for BP control (Table 2). Patients with a diagnosis of hypertension at recruitment were less likely to have their BP controlled at 6 months for each of the targets outlined.

Blood pressure in right versus left arms A reading was unavailable from both arms in 18 (7\%) patients. Of patients with available readings bilaterally, 27 patients (11.3\%) had a discrepancy of at least $20 \mathrm{mmHg}$ between systolic BP readings, an indicator of high risk for future cardiovascular events [19].

Ambulatory blood pressure monitoring A $24 \mathrm{~h}$ ambulatory BP monitor was applied to 210 patients (82\% of participants). Thirteen patients did not wear their monitor overnight, likely due to device intolerance. Mean $24 \mathrm{~h} \mathrm{BP}$ was $127 / 73 \mathrm{mmHg}$ (systolic SD 15.6 \& range 90-222; diastolic SD 9.6 \& range 53-116). Daytime mean was 128/74 (systolic SD 15.7 \& range 91-222; diastolic SD 10 \& range 53-116) and nightime mean was 121/68 (systolic SD 15.1 \& range 88-176; diastolic SD 9.7 \& range 48-95). When compared with ESH Guidelines [20], more patients had their BP controlled by day (target $135 / 85 ; 61.4 \%$ ) than by night (target 120/70; $43.6 \%)$. Fifty-nine percent had overall $24 \mathrm{~h} \mathrm{BP}$ control at target $(130 / 80)$.

\section{Waist circumference and BMI}

The mean waist circumference was $90 \mathrm{~cm}$ for females $(n=95)$ and $97 \mathrm{~cm}$ for males $(n=142)$. Two-thirds

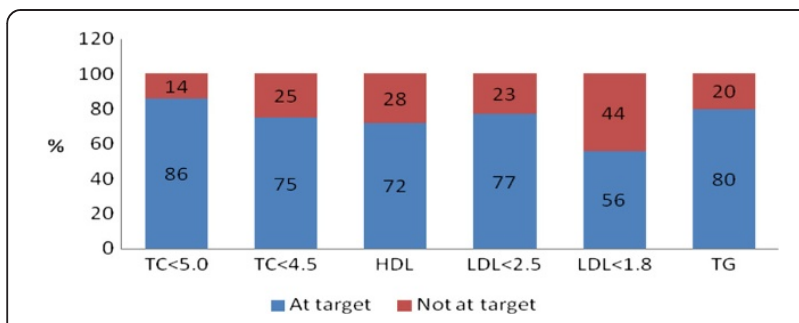

Fig. 1 Proportions of patients with lipid results at target at 6 months; $\mathrm{TC}=$ total cholesterol (targets $<5.0 \mathrm{mmol} / \mathrm{L}$ or $<4.5 \mathrm{mmol} / \mathrm{L}$ (high-risk target) [15]); $\mathrm{HDL}=$ high-density lipoprotein (target $>1$ in males and $>1.2 \mathrm{mmol} / \mathrm{L}$ in females); $\mathrm{LDL}=$ low-density lipoprotein (targets $<2.5 \mathrm{mmol} / \mathrm{L}$ or $<1.8 \mathrm{mmol} / \mathrm{L}$ (high-risk target) [15]); TG = triglycerides (target $<1.7 \mathrm{mmol} / \mathrm{L}$ )
Table 2 Proportion of patients with BP at target (total $N=249$ )

\begin{tabular}{llccc}
\hline \multirow{5}{*}{ BP target } & \multicolumn{4}{l}{ Proportion of patients at target } \\
\cline { 2 - 5 } & All patients & $\begin{array}{l}\text { With diagnosed } \\
\text { hypertension }\end{array}$ & $\begin{array}{l}\text { No hypertension } \\
\text { diagnosed }\end{array}$ & $P$-value \\
\hline$\leq 130 / 80^{\text {a }}$ & $40(16.1 \%)$ & $15(10.5 \%)$ & $25(23.6 \%)$ & $p=0.005$ \\
$\leq 140 / 90^{b}$ & $91(36.6 \%)$ & $43(30.1 \%)$ & $48(45.3 \%)$ & $p<0.05$ \\
$\leq 135 / 85^{c}$ & $69(27.7 \%)$ & $31(21.7 \%)$ & $38(35.6 \%)$ & $p<0.05$ \\
\hline
\end{tabular}

$B P$ blood pressure

${ }^{\text {a } T a r g e t ~ f r o m ~ E u r o p e a n ~ g u i d e l i n e s ~ o n ~ c a r d i o v a s c u l a r ~ d i s e a s e ~ p r o t e c t i o n ~}$ (high-risk patients) [15] \& UK RCP National Clinical Guideline on Stroke [5] \& ESH Guideline 2007 [20] (after stroke)

${ }^{\mathrm{b}}$ Target used in EUROASPIRE studies [9]

'Target in IHF guideline on stroke [6]; $p$-values apply to comparisons between those with and without diagnosis of hypertension

(67\%) had a waist circumference above target, including $79 \%$ of females and $58 \%$ of males. The mean BMI in 250 patients was 26.8 (range 17-48; SD 4.5). The proportions of patients classified as 'normal weight (18.5$\left.25 \mathrm{~kg} / \mathrm{m}^{2}\right)$ ', 'overweight $\left(25-30 \mathrm{~kg} / \mathrm{m}^{2}\right)$ ' and 'obese $>30 \mathrm{~kg} / \mathrm{m}^{2}$ )' were 32,44 and $24 \%$ respectively.

\section{Smoking}

The proportion of patients (M:F, 60:40) that smoked 6 months post stroke was $16.4 \%(n=42)$, compared with $28 \%(n=72)$ at baseline.

\section{Secondary preventive therapy Antithrombotic medications}

Almost all (249; $97.3 \%)$ were on an anti-platelet and/or anticoagulant medication. 160 patients $(62.5 \%)$ were on anti-platelet medication and, of these, 36 patients $(22.5 \%)$ were on dual anti-platelet therapy. Patients with carotid artery disease were significantly more likely to be on dual therapy ( $41 \%$ versus $9 \%, p<0.001) .116$ patients $(45.3 \%)$ were on anti-coagulation therapy. Of those on Warfarin $(n=100)$, the mean INR was $2.4(1.2-3.7) .78 \%$ had an INR between 2 and $3.14 \%$ had a sub-therapeutic INR and the remaining $8 \%$ had a supra-therapeutic INR level. One quarter of patients on anti-coagulation $(n=28 ; 24 \%)$ were also on anti-platelet therapy. Of patients with atrial fibrillation $(N=97), 83.5 \%(81 / 97)$ were on anticoagulation at 6 months. The remaining patients (16/97; $16.5 \%)$ were not on anticoagulation due to a contra-indication (12), patient too unwell (3) or patient refusal (1).

\section{Lipid-lowering and anti-hypertensive medications}

At follow-up, almost all patients (242; $94.5 \%)$ were on lipid-lowering therapy and three-quarters of patients ( $N=190 ; 74.2 \%)$ were on anti-hypertensive therapy. Almost half of these patients were on single agent therapy, with a further one-third (33\%) on dual anti-hypertensive therapy. Remaining patients were on three $(32 ; 16.8 \%)$ or four $(5 ; 2.5 \%)$ agents. Those most commonly prescribed anti-hypertensives were beta blockers $(N=101,39.5 \%)$, 
angiotensin-converting-enzyme inhibitors $(N=95,37.1 \%)$ and calcium channel blockers $(N=55,21.5 \%)$. Although patients on lipid-lowering therapy were more likely to reach lipid targets, patients on anti-hypertensive therapy had poor therapeutic control of blood pressure at 6 months (Table 3).

\section{Discussion}

This study reveals suboptimal control of many risk factors post IS despite the availability of evidence-based therapies and clear guidelines for secondary prevention. This cohort was at high-risk for recurrent stroke, with prevalent baseline risk factors, two-thirds over the age of 65 years, and one-quarter having previous stroke. These findings raise concern for health professionals and should prompt more intensive management of risk factors.

Studies have shown an association between TC, LDL and elevated TG with IS risk, especially among atherosclerotic and lacunar stroke subtypes [21]. Although mean lipid levels recorded at 6 months were not excessively high, many results were not at target. Only three-quarters of our cohort had TC at target $(<4.5 \mathrm{mmol} / \mathrm{L})$ with a similar proportion (77 \%) having LDL at target $(<2.5 \mathrm{mmol} / \mathrm{L})$. However, these results compare favourably with those from EUROASPIRE III (49 and $55 \%$ respectively at target) [8] and with results from the stroke-specific module of EUROASPIRE III [10], likely due to the higher uptake of lipid-lowering medications in our group. Outside the trial setting, there is currently no published data available on the adequacy of control of lipid levels specific to IS. Although the optimal LDL level for secondary stroke prevention is unclear, there is evidence from the SPARCL study that tight control $(<1.8 \mathrm{mmol} / \mathrm{L}$; achieved by only $44 \%$ of our cohort) reduces recurrent IS risk by one quarter [22] and European guidelines now advise this target for high-risk patients [15].
Diabetes is independently related to a greater risk of IS (adjusted risk ratio, 2.26) [23]. For diabetic patients in ASPIRE-S with suboptimal control (HbA1c $\geq 7,28 \%$ ), it is unknown (without access to patient notes at followup) whether the treating physician opted for less strict control in light of poorly tolerated hypoglycaemic episodes in some patients. Optimal target HbA1c levels in diabetic patients still remain the subject of much debate, and in most cases is individualised to particular patient characteristics [24]. Only $1.5 \%$ of non-diabetic patients had FG $\geq 7 \mathrm{mmol} / \mathrm{L}$. Four percent of patients had FG within the impaired fasting glucose range $(\geq 6.1 \mathrm{mmol} /$ $\mathrm{L})$, but this rose to almost $10 \%$ when the latest American Diabetes Association definition ( $\geq 5.6 \mathrm{mmol} / \mathrm{L})$ was applied [16]. Diagnoses were not made on the basis of a single FG level in ASPIRE-S but it is known that early dietary and lifestyle modifications can delay or prevent the development of diabetes in patients with impaired FG [25].

The prevalence of obesity in the developed world has substantially increased over time [26]. Results from the Physician's Health Study reported a significant increase in the relative risk of stroke with each unit increase of BMI, independent of the effects of other stroke risk factors [27]. Over two-thirds of our cohort had a BMI of $\geq 25 \mathrm{~kg} / \mathrm{m}^{2}$ with one quarter being classified as obese. These levels are higher than those reported in the general Irish population (overweight $39 \%$ and obese $18 \%$ ) [28] and likely contribute to other stroke/cardiovascular risk factors such as hypertension, hyperlipidaemia and diabetes. Two-thirds of participants had abdominal obesity. These results suggest that a more active role for sustained professional support and motivation in achieving successful weight reduction post stroke is needed, including (repeated) clear advice on weight loss from health professionals, which positively impacts on weight

Table 3 Therapeutic control of lipids and office blood pressure at follow-up

\begin{tabular}{|c|c|c|c|c|}
\hline & \multicolumn{4}{|c|}{ Proportion reaching target (\%) } \\
\hline & All patients & On lipid-lowering medication & No lipid-lowering treatment & $p$-value \\
\hline \multicolumn{5}{|c|}{ Lipid targets ${ }^{\mathrm{a}}(\mathrm{mmol} / \mathrm{l})$} \\
\hline $\mathrm{TC}<5.0$ & (200) 86.2 & 89.5 & (4) 30.8 & $p<0.001$ \\
\hline $\mathrm{TC}<4.5$ & (174) 75.0 & (171) 78.1 & (3) 23.1 & $p<0.001$ \\
\hline $\mathrm{LDL}<2.5$ & (170) 76.9 & (167) 79.5 & (3) 27.3 & $p<0.001$ \\
\hline \multirow[t]{2}{*}{$\mathrm{LDL}<1.8$} & (97) 43.9 & (96) 45.7 & (1) 9.1 & $p<0.05$ \\
\hline & All patients & On anti-hypertensive medication & No anti-hypertensive medication & $p$-value \\
\hline \multicolumn{5}{|c|}{ BP targets $(\mathrm{mmHg})$} \\
\hline$B P<140 / 90$ & (91) 36.6 & (62) 33.2 & (29) 46.8 & $P=0.05$ \\
\hline $\mathrm{BP}<135 / 85$ & (69) 27.7 & (46) 24.6 & (23) 37.1 & $P=0.06$ \\
\hline$B P<130 / 80$ & (40) 16.1 & (24) 12.8 & (16) 25.8 & $p<0.05$ \\
\hline
\end{tabular}

TC total cholesterol, LDL low-density lipoprotein, BP blood pressure

${ }^{a}$ European Guidelines on cardiovascular disease prevention (version 2012) [15] 
loss behaviour [29]. Although multiple successful weight reduction interventions are available, the ability of patients post stroke to engage in vigorous exercise programmes may be limited. Studies to explore the effectiveness of interventions such as group education or dietary changes for weight loss specifically after stroke are limited. The first randomized-controlled trial to evaluate the efficacy and safety of a weight management intervention in stroke survivors using the System$\mathrm{CHANGE}^{\mathrm{mm}}$ approach is currently underway [30].

The INTERSTROKE study reported a doubling in the risk for IS in current smokers (OR 2.09, 1.75-2.51) [3]. Although smoking rates are reportedly decreasing over time [2], $16 \%$ were still smoking in our cohort at 6 months. This reflects a smoking cessation rate of $41 \%$ (compared with $81 \%$ in the Irish subgroup of EUROASPIRE III and $40 \%$ in the stroke-specific module) [8, 10]. Although physician advice to stop smoking is the most important first step in the cessation process, this advice must be reiterated and reinforced by all health professionals [31]. Multiple pharmacotherapies for tobacco dependence have been shown to increase smoking cessation rates [32], and behavioural interventions can further increase the success of smoking cessation [33].

Although the mean BP reading was not excessively elevated, only $37 \%$ of patients had $\mathrm{BP} \leq 140 / 90$ (similar to EUROASPIRE stroke module; 38 \%). Such poor control of BP may reflect a lack of focus on secondary preventive initiatives for patients after stroke. The proportion at target was lower amongst patients with a previous diagnosis of hypertension and amongst those on antihypertensive therapy, reflecting the difficulty in treating hypertension in clinical practice. Recent European guidelines $[5,15]$ suggest a lower BP target of $\leq 130 / 80$ in patients after IS, where tolerated. Only $16 \%$ of our cohort achieved this target at 6 months and this proportion dropped to $13 \%$ amongst those on anti-hypertensive therapy. Although these results reflect a single reading in patients, they are a reminder that better management of BP is required after IS including greater up-titration of medication dosages and use of multiple medication regimens in addition to lifestyle advice.

Ambulatory BP monitoring (reflecting multiple measurements) is known to provide a more accurate measure of BP control [34]. This is one of the first studies to describe ambulatory BP control post acute stroke and results clearly demonstrate greater overall BP control, compared with office readings. Better control of daytime (versus nightime) readings may reflect the timing of administration of antihypertensive medications (usually in the morning) which impacts on circadian control [35]. It may also reflect lack of attention placed by physicians on nightime BP control. Nightime BP control has been strongly linked to an increased risk of cardiovascular disease in multiple studies, including the Dublin outcome study of 5292 participants [36], which reported a relative hazard ratio (for cardiovascular mortality) of 1.21 for each $10-\mathrm{mm} \mathrm{Hg}$ increase in nightime systolic BP. This increased risk remained significant in older patients [36, 37].

Most IS patients are prescribed a combination of cardiovascular medications and this multi-factorial approach to secondary stroke prevention can result in a substantial reduction (of up to $80 \%$ ) in future stroke risk [38]. Although prescription rates of anti-thrombotic and lipid-lowering medications in our cohort were high, the prescription of anti-hypertensive medications appeared suboptimal. Despite positive findings from the pivotal PROGRESS study [39], a minority of patients in ASPIRE-S were on dual ACE I and diuretic therapy. However, a significant proportion of patients had hypertension (58\%), atrial fibrillation ( $40 \%$ ) and/or heart disease $(30 \%)$ at baseline and were likely already established on appropriate medications with anti-hypertensive effects (such as beta-blockers or CCBs) prior to their stroke.

This study has many strengths including its prospective design, large sample size, high rate of follow-up, inhospital assessment of patients to verify eligibility for inclusion and a standardised follow-up assessment at 6 months modelled on previous robust EUROASPIRE surveys. There is little information in the stroke literature on the adequacy of secondary prevention after IS and much of the findings from ASPIRE-S are therefore novel. The recent EUROASPIRE III stroke-specific module (including four countries) reported some comparable findings but was retrospective, excluded IS patients over 80 , had a broader follow-up period (6-36 months), used self-reported definitions of vascular risk factors and unhealthy lifestyle habits, did not include ambulatory BP monitoring and had limited generalizability.

\section{Limitations}

The majority of the findings from ASPIRE-S are novel within the Irish stroke setting (and further afield), but there are some limitations to this study. Participants were recruited over 12 months as a representative (not consecutive) sample of ischaemic stroke patients in North Dublin. However, no attempt was made to be selective in any way and this cohort compares favourably in many ways with that recruited in 2006 as part of the North Dublin Population Stroke Study (NDPSS) [11]. Although the ASPIRE-S sample of three hospitals reflects the north Dublin population well, results may not be representative of the population nationally. It may be that the burden of cardiovascular risk factors is particularly high in north Dublin. Higher prevalence of cardiovascular risk factors has previously been linked with social disadvantage $[40,41]$ and the north Dublin population 
includes several communities where social disadvantage is common. Furthermore, although ASPIRE-S has a large overall sample size, the statistical significance of some results may have been limited by small numbers within subcohorts.

\section{Conclusion}

Given the strong evidence from consensus of expert opinion for multi-dimensional risk factor management in secondary stroke prevention [38] it is imperative that tailored programmes of care (including risk factor control) be optimised. These should commence during the patients' hospital admission with seamless transfer to the community. Over recent years there have been significant advances in the standardisation of clinical guidelines for stroke care, however the introduction of national stroke clinical guidelines alone is insufficient to improve health care quality [42]. For efficacy, guidelines should be incorporated into quality assurance cycles with education programmes and local feedback [43]. Results from ASPIRE-S promote awareness of the importance of ongoing surveillance of cardiovascular risk in patients after IS and prompt further roll-out of similar surveys internationally. This should encourage the establishment of local policies which support comprehensive, professional, multi-disciplinary secondary preventive initiatives, accessible to all survivors of stroke.

\section{Competing interests}

The authors declare that they have no competing interests.

\begin{abstract}
Authors' contributions
$L B$ is lead author on this paper. $L B, L M$ and PH collected, cleaned and analysed the data for ASPIRE-S. LM and PH revised the draft paper. DW and AH designed the study and data collection tools, implemented the study, monitored data collection and revised the paper. ED, FH and ES had input into study design and implementation and revised the draft paper. All authors read and approved the final manuscript.
\end{abstract}

\section{Acknowledgements}

We acknowledge the ASPIRE-S Steering Group members: Professor Hannah McGee, Royal College of Surgeons in Ireland and Professor Peter Kelly, Mater Misericordiae University Hospital, Dublin. The ASPIRE-S research was supported by a Health Research Award from the Irish Health Research Board (HRB; grant number 1404/7400).

\section{Author details}

${ }^{1}$ Department of Geriatric \& Stroke Medicine, Royal College of Surgeons in Ireland (RCSI), Beaumont Hospital, Dublin 9, Ireland. ${ }^{2}$ Department of Psychology, RCSI, Dublin 2, Ireland. ${ }^{3}$ Department of Geriatric \& Stroke Medicine, Connolly Hospital, Blanchardstown, Dublin 15, Ireland. ${ }^{4}$ School of Physiotherapy, RCSI, Dublin 2, Ireland. ${ }^{5}$ Department of Epidemiology and Public Health, RCSI, Dublin 2, Ireland.

Received: 8 May 2015 Accepted: 7 October 2015

Published online: 23 October 2015

\section{References}

1. Feigin VL, Forouzanfar MH, Krishnamurthi R, Mensah GA, Conor M, Bennett DA, et al. Global and regional burden of stroke during 1990-2010: findings from the Global Burden of Disease Study 2010. Lancet. 2014;383:245-54.

2. Rothwell PM, Coull AJ, Giles MF, Howard SC, Silver LE, Bull LM, et al. Change in stroke incidence, mortality, case-fatality, severity, and risk factors in
Oxfordshire, UK from 1981 to 2004 (Oxford Vascular Study). Lancet. 2004;363:1925-33.

3. O'Donnell MJ, Xavier D, Liu L, Zhang H, Chin SL, Rao-Melacini P, et al. Risk factors for ischaemic and intracerebral haemorrhagic stroke in 22 countries (the INTERSTROKE study): a case-control study. Lancet. 2010;376:112-23.

4. Wolf PA, D'Agostino RB, Belanger AJ, Kannel WB. Probability of stroke: a risk profile from the Framingham Study. Stroke. 1991;22:312-8.

5. National Clinical Guideline for Stroke. London: Royal College of Physicians UK 2012. https://www.rcplondon.ac.uk/sites/default/files/national-clinicalguidelines-for-stroke-fourth-edition.pdf. Acessed 29th November 2014.

6. National Clinical Guidelines for the Care of People with Stroke and TIA: Irish Heart Foundation 2013. http://www.rishheart.ie/media/pub/strokereports/ FinalMarch2010.pdf. Accessed 3rd March 2013.

7. EUROASPIRE II Study Group. Lifestyle and risk factor management and use of drug therapies in coronary patients from 15 countries; principal results from EUROASPIRE II Euro Heart Survey Programme. Eur Heart J. 2001;22:554-72.

8. Kotseva K, Wood D, De Backer G, De Bacquer D, Pyorala K, Keil U. EUROASPIRE III: a survey on the lifestyle, risk factors and use of cardioprotective drug therapies in coronary patients from 22 European countries. Eur J Cardiovasc Prev Rehabil. 2009;16:121-37.

9. Kotseva K, Wood D, De Backer G, De Bacquer D, Pyorala K, Keil U. Cardiovascular prevention guidelines in daily practice: a comparison of EUROASPIRE I, II, and III surveys in eight European countries. Lancet. 2009;373:929-40,

10. Heuschmann PU, Kircher J, Nowe T, Dittrich R, Reiner Z, Cifkova R, et al. Control of main risk factors after ischaemic stroke across Europe: data from the stroke-specific module of the EUROASPIRE III survey. Eur J Prev Cardiol. 2014 [published online ahead of print August 19, 2014].

11. Kelly PJ, Crispino G, Sheehan O, Kelly L, Marnane M, Merwick A, et al. Incidence, event rates, and early outcome of stroke in Dublin, Ireland: the North Dublin population stroke study. Stroke. 2012;43(8):2042-7.

12. Marnane M, Duggan CA, Sheehan OC, Merwick A, Hannon N, Curtin D, et al. Stroke subtype classification to mechanism-specific and undetermined categories by TOAST, A-S-C-O, and causative classification system: direct comparison in the North Dublin population stroke study. Stroke. 2010;41(8):1579-86.

13. Adams Jr HP, Bendixen BH, Kappelle LJ, Biller J, Love BB, Gordon DL, et al. Classification of subtype of acute ischemic stroke. Definitions for use in a multicenter clinical trial. TOAST. Trial of Org 10172 in Acute Stroke Treatment. Stroke. 1993;24:35-41.

14. Bamford J, Sandercock P, Dennis M, Burn J, Warlow C. Classification and natural history of clinically identifiable subtypes of cerebral infarction. Lancet. 1991;337:1521-6.

15. Perk J, De Backer G, Gohlke H, Graham I, Reiner Z, Verschuren M, et al. European Guidelines on cardiovascular disease prevention in clinical practice (version 2012). The Fifth Joint Task Force of the European Society of Cardiology and Other Societies on Cardiovascular Disease Prevention in Clinical Practice. Eur Heart J. 2012;33:1635-701.

16. American Diabetes Association. Standards of Medical Care in Diabetes_-2013. Diabetes Care. 2013;36:S11-66.

17. Clinical guidelines on the identification, evaluation, and treatment of overweight and obesity in adults: executive summary. Expert panel on the identification, evaluation, and treatment of overweight in adults. Am J Clin Nutr. 1998;68(4):899-917.

18. Genuth S, Alberti KG, Bennett P, Buse J, Defronzo R, Kahn R, et al. Expert Committee on the Diagnosis and Classification of Diabetes Mellitus: Followup report on the diagnosis of diabetes mellitus. Diabetes Care. 2003:26:3160-7.

19. Clark CE, Taylor RS, Shore AC, Ukoumunne OC, Campbell JL. Association of a difference in systolic blood pressure between arms with vascular disease and mortality: a systematic review and meta-analysis. Lancet. 2012;379:905-14.

20. Mancia G, De Backer G, Dominiczak A, Cifkova R, Fagard R, Germano G, et al. 2007 Guidelines for the Management of Arterial Hypertension: The Task Force for the Management of Arterial Hypertension of the European Society of Hypertension (ESH) and of the European Society of Cardiology (ESC). J Hypertens. 2007;25:1105-87.

21. Ovbiagele B, Nguyen-Huynh MN. Stroke epidemiology: advancing our understanding of disease mechanism and therapy. Neurotherapeutics. 2011;8(3):319-29

22. Amarenco P, Goldstein LB, Szarek M, Sillesen H, Rudolph AE, Callahan A, et al. Effects of intense low-density lipoprotein cholesterol reduction in 
patients with stroke or transient ischemic attack: the Stroke Prevention by Aggressive Reduction in Cholesterol Levels (SPARCL) trial. Stroke. 2007:38:3198-204.

23. Folsom AR, Rasmussen ML, Chambless LE, Howard G, Cooper LS, Schmidt $\mathrm{Ml}$, et al. Prospective associations of fasting insulin, body fat distribution, and diabetes with risk of ischemic stroke. The Atherosclerosis Risk in Communities (ARIC) Study Investigators. Diabetes Care. 1999;22(7):1077-83.

24. Inzucchi SE, Bergenstal RM, Buse JB, Diamant M, Ferrannini E, Nauck M, et al. Management of hyperglycaemia in type 2 diabetes: a patient-centered approach. Position statement of the American Diabetes Association (ADA) and the European Association for the Study of Diabetes (EASD). Diabetologia. 2012;55(6):1577-96.

25. Nathan DM, Davidson MB, DeFronzo RA, Heine RJ, Henry RR, Pratley R, et al. Impaired fasting glucose and impaired glucose tolerance: implications for care. Diabetes Care. 2007;30(3):753-9.

26. Caballero B. The global epidemic of obesity: an overview. Epidemiol Rev. 2007;29:1-5

27. Kurth T, Gaziano JM, Berger K, Kase CS, Rexrode KM, Cook NR, et al. Body mass index and the risk of stroke in men. Arch Intern Med. 2002;162:2557-62.

28. Report of the National Taskforce on Obesity: Obesity - the policy challenges. Irish Department of Health website. 2005. http://www.hse.ie/eng/health/ child/healthyeating/taskforceonobesity.pdf. Accessed 2013 1st August.

29. Jackson SE, Wardle J, Johnson F, Finer N, Beeken RJ. The impact of a health professional recommendation on weight loss attempts in overweight and obese British adults: a cross-sectional analysis. BMJ Open. 2013;3(11):e003693.

30. Plow M, Moore SM, Kirwan JP, Frost F, Katzan I, Jaeger S, et al. Randomized controlled pilot study of a SystemCHANGE weight management intervention in stroke survivors: rationale and protocol. Trials. 2013;14:130.

31. Lancaster T, Stead L. Physician advice for smoking cessation. Cochrane Database Syst Rev. 2004;4:CD000165.

32. Mills EJ, Wu P, Spurden D, Ebbert JO, Wilson K. Efficacy of pharmacotherapies for short-term smoking abstinance: a systematic review and meta-analysis. Harm Reduct J. 2009;6:25.

33. Stead LF, Lancaster T. Combined pharmacotherapy and behavioural interventions for smoking cessation. Cochrane Database Syst Rev. 2012;10:CD008286.

34. O'Brien E. Ambulatory blood pressure monitoring in the management of hypertension. Heart. 2003;89:571-6.

35. Minutolo R, Gabbai FB, Borrelli S, Scigliano R, Trucillo P, Baldanza D, et al. Changing the timing of antihypertensive therapy to reduce nocturnal blood pressure in CKD: an 8-week uncontrolled trial. Am J Kidney Dis. 2007;50:908-17.

36. Dolan E, Stanton A, Thijs L, Hinedi K, Atkins N, McClory S, et al. Superiority of ambulatory over clinic blood pressure measurement in predicting mortality: the Dublin outcome study. Hypertension. 2005;46:156-61

37. Burr ML, Dolan E, O'Brien EW, O'Brien ET, McCormack P. The value of ambulatory blood pressure in older adults: the Dublin outcome study. Age Ageing. 2008;37:201-6.

38. Hackam DG, Spence JD. Combining multiple approaches for the secondary prevention of vascular events after stroke: a quantitative modeling study. Stroke. 2007:38:1881-5.

39. PROGRESS Collaborative Group. Randomised trial of a perindopril-based blood-pressure-lowering regimen among 6,105 individuals with previous stroke or transient ischaemic attack. Lancet. 2001;358(9287):1033-41.

40. Fitzsimon N, Johnson H, Hayes C, Mulholland D, Kelleher CC. Predictors of poor self-rated health at area level in the Eastern Regional Health Authority: a multilevel analysis. Ir Med J. 2007;100(8):suppl 47-9.

41. Kelleher CC, Friel S, Nic Gabhainn S, Tay JB. Socio-demographic predictors of self-rated health in the Republic of Ireland: findings from the national survey on lifestyle, attitudes and nutrition, SLAN. Soc Sci Med. 2003;57(3):477-86.

42. Donnellan C, Sweetman S, Shelley E. Implementing clinical guidelines in stroke:A qualitative study of perceived facilitators and barriers. Health Policy. 2013;111:234-44

43. Donnellan C, Sweetman S, Shelley E. Health professionals' adherence to stroke clinical guidelines: a review of the literature. Health Policy. 2013;111(3):245-63.

\section{Submit your next manuscript to BioMed Central and take full advantage of:}

- Convenient online submission

- Thorough peer review

- No space constraints or color figure charges

- Immediate publication on acceptance

- Inclusion in PubMed, CAS, Scopus and Google Scholar

- Research which is freely available for redistribution

Submit your manuscript at www.biomedcentral.com/submit 